not active in the main sequence, especially if all stars have dense hot cores, as Milne believes. Thus the very latest stages of degeneracy, where the specific heat is small and contraction difficult, must be run through rapidly, and the total duration of the white dwarf stage probably is not much greater than we have already calculated. The probable relative abundance of white dwarfs is then somewhat difficult to reconcile with a time-scale of $10^{13}$ or $10^{14}$ years, but fits well with the transmutation theory time-scale.

The view that the nuclei of planetary nebulæ are 'white dwarfs' has, we now find, already been propounded by Jeans in "The Universe Around Us", pp. 309-311. That we had both failed to notice this can be excused, if at all, only by the great popularity of the publication in which the theory was announced, but we sincerely regret the oversight. In the application of this result we differ from Jeans, since he tentatively placed these stars at the beginning of stellar evolution, while we place them, with the other white dwaris, at the end.

$$
\begin{aligned}
& \text { Princeton University. } \\
& \text { H. N. Russelt. } \\
& \text { Rutgers University, } \\
& \text { Mar. } 31 . \\
& 1 \text { Zeit. f. Astrophysik, 2, p. 1; } 1931 . \\
& \begin{array}{l}
2 \text { Cf. Russell, Dugan, and stewart, “Astronomy ”, p. } 835 . \\
3 \text { Mon. Not. R.A.S.,91, p. } 39 ; 1930 .
\end{array} \\
& \text { R. D'E. Atrkinson. }
\end{aligned}
$$

\section{Emission Bands in the Mercury Spectrum under Low Excitation.}

Some years ago I found a long series of diffuse bands from $\lambda 2943$ to $\lambda 2614$ in the absorption spectrum of a long column of mercury vapour. ${ }^{1}$ The same series was found independently and about the same time by Mohler and Moore.

Up to the present, these bands have never been obtained in emission. I now find that when mereury vapour is fluorescing under the iron are (excitation from $\lambda 2650$ to $\lambda 2537$ ) these bands are emitted, and in much greater intensity if the vapour is superheated. The continuous emission from about $\lambda 2950$ to $\lambda 3600$ (maximum at $\lambda 3300$ ) is also much increased by superheating, while the green visual fluorescence is extinguished. So far as my experiments go, the emission bands seem to be in close relation to this continuous emission at $\lambda 3300$, though I am not prepared at present to say that this is invariably the case. Much longer exposures are required for the bands than for $\lambda 3300$, but when such exposures are given, the bands appear as a kind of prolongation of the continuous emission. The continuous region, it is to be noted, is at the less refrangible end of the band series, where the band spacing is widest, and not beyond the convergence point, and this continuous region, unlike the bands, is not known in absorption. It will be important to examine the absorption of the superheated vapour.

The remarkable phenomena connected with the band spectra of mercury, and the long time of duration of the emission, are very complicated, and I think that speculation has rather outrun our knowledge of the facts, which is by no means adequate. It is my aim to find out as much as possible under conditions of low frequency excitation, less than the frequency of the atomic resonance line $\lambda 2537$. These conditions should reduce the problem to its simplest form.

Terling Place, Chelmsford, RAYLEIGH. April 20.

${ }^{1}$ Proc. Roy. Soc., A, vol. 116, p. 705; 1927.

No. 3209, VoL. 127]

\section{Proton and Electron.}

THE ratio of the mass of the proton, $M_{P}$, to that of the electron, $m_{e}$, is a pure number which is likely to be of considerable importance in physical theory. In a recent letter, Dr. W. N. Bond ${ }^{1}$ has deduced for this ratio the value $1846 \cdot 5_{7} \pm 0 \cdot 4_{8}$ with a probable error which should be accurate to about ten per cent. This is very close to the 'deflection' value of Birge," $1846 \cdot 61 \pm 2$, but larger than the 'spectroscopic' value $1838 \cdot 26 \pm 1$.

The suggestion of A. P. Mathews that

$$
\frac{M_{P}}{m_{e}}=\left(\frac{135}{\pi}\right)^{2}=1846 \cdot 58
$$

gives remarkable numerical concordance with Bond's estimate, but it seems, at present, to have no theoretical significance.

As Dr. Bond has pointed out, the relation proposed by Rojansky ${ }^{3}$ and also by Eddington, ${ }^{4}$ which gives

$$
\frac{M_{P}}{m_{e}}=\frac{(136)^{2}}{10}=1849 \cdot 6,
$$

is not in good agreement with recent experimental determinations.

The same objection applies to Witmer's hypothesis ${ }^{5}$ that

$$
\frac{M_{P}}{m_{e}}=(43)^{2}=1849 \text {. }
$$

I venture to add one more suggestion to the many already made as to the ratio of these two masses. Fürth ${ }^{6}$ has obtained a remarkable relation involving the constant of gravitation, $G$, and the mass of the 'neutron' which he regards as formed by the combination of a positive and negative electron. Assuming this mass to be the same as that of the hydrogen atom, $M_{H}$, his relation may be written in the simplified form $2 b c / G M_{H}{ }^{2}=(16)^{32}$ where $b=h / 2 \pi$. This gives good agreement with experimental results.

The power of 16 which occurs in Fürth's relation is introduced in considering the number of arrangements in a 16-dimensional continuum as in Edding. ton's theory, which requires 16 parameters for the complete determination of an electron. For an electron Fürth finds a total of $16^{16}$ possible arrangements and for a 'neutron' $16^{32}$ distinct possibilities. $H$ is formula suggested to me that the ratio of the masses of proton and electron might be of the form

$$
\frac{M_{P}}{m_{e}}=\left(\frac{16}{10}\right)^{16}=1844 \cdot 68 .
$$

Here again, the number 16 represents the number of parameters or ' degrees of freedom' which, according to Proca, ${ }^{7}$ are characteristic of an electron. These Proca classifies as 4 co-ordinates, 4 moments, 3 components of magnetic moment, 3 components of electric moment, the mass, and the de Broglie wavelength. The presence of the integer 10 in the above relation may be explained by assuming that, of the 16 degrees of freedom associated with an electron, 6 are suppressed or 'frozen ' in the case of the positive electron. It is natural to suppose that these are the three components of the electric moment and the three components of the magnetic moment, all of which are here regarded as having some real physical meaning for the negative electron. Perhaps the proton, as contrasted with the negative electron, is simply 'a hole in the ether' without electric or magnetic moment. This hypothesis reverses the rôles assigned to these two entities in 1913 by S. B. McLaren, who assumed that the positive electron was the magneton.

It is with some hesitation that I put forward this tentative suggestion, partly because the theory of 